\title{
NETS OF SUBGROUPS AND AMENABILITY
}

\section{SCHOCHETMAN}

ABstract. We first investigate some convergence properties of nets in the topological space of closed subgroups of a locally compact group. We then use these properties to answer some questions concerning the amenable subgroups.

Introduction. Let $G$ be a locally compact group and $\mathscr{K}(G)$ the set of all closed subgroups of $G$ equipped with the compact-open topology (see $\$ 1$ for the definition). If $H$ is a closed (resp. closed normal) subgroup of $G$, then there is a canonical correspondence between the elements of $\Re(G)$ contained in (resp. containing) $H$ and the elements of $\mathscr{K}(H)$ (resp. $\mathscr{K}(G / H)$ ). In $\S 2$ we show these are homeomorphisms. We then give necessary and sufficient conditions for a net in $\mathscr{K}(G)$ directed by set containment (resp. inclusion) to converge to $G$ (resp. the trivial subgroup).

In $\$ 3$ we consider the collection of groups in $\Re(G)$ which are amenable. We give sufficient conditions for the limit of a net of amenable subgroups to be amenable and another condition which is equivalent to amenability of $G$. Finally, if $G$ is not amenable, then it does not contain a largest amenable subgroup; however, it does contain a maximal one.

1. Preliminaries. Recall $[5$, p. 427] that a basic open subset in the compact-open topology of $\mathfrak{K}(G)$ is of the form $\mathfrak{u}(C, \mathfrak{F})=\{K \in \mathfrak{K}(G)$ : $K \cap C=\varnothing, K \cap B \neq \varnothing$, for each $B \in \mathcal{F}\}$, where $C$ is a compact subset of $G$ and $\mathcal{F}$ is a finite family of nonempty open subsets of $G$. (Note that $C$ and $\mathscr{F}$ may be empty.) With this topology, $\mathscr{K}(G)$ is a compact Hausdorff space. Let $\delta(G)$ denote the space of subgroup representations of $G$, i.e. pairs $(K, T)$ where $K \in \mathcal{K}(G)$ and $T$ is a (unitary equivalence class of a) unitary representation of $K$. We assume $\delta(G)$ to be equipped with its usual inner hull-kernel topology $[5, \mathrm{p}$. 249]. $E$ will denote the trivial subgroup $\{e\}$ of $G$ and 1 its identity representation (it is also the regular representation). Let $R$ (resp. $I$ ) denote the regular (resp. identity) representation of $G$. If $\left\{K_{i}\right\}$ is a net in $\mathfrak{K}(G), I_{i}$ (resp. $R_{i}$ ) will denote the identity (resp. regular) representation of $K_{i}$. If $H, K \in \Re(G)$ with $K \supseteq H$ and $T$ a representa-

Received by the editors July 20, 1970.

AMS 1970 subject classifications. Primary 22D05; Secondary 22D10, 22D30.

Key words and phrases. Locally compact group, space of closed subgroups, net of subgroups, amenable group, weak containment, subgroup representation, induced representation. 
tion of $H$, then ${ }_{K} U^{T}$ will denote the usual induced representation of $K$ (see [7, p. 312] or [2]). In particular, if $K=K_{i}$ as above (resp. $K=G)$, then we write ${ }_{i} U^{T}$ (resp. $\left.U^{T}\right)$. We will use $K$ to denote a generic element of $\mathcal{K}(G)$ and $J$ (resp. $P$ ) its identity (resp. regular) representation. We also assume the reader is familiar with the notions of amenability [6] and weak containment [3].

LEMMA 1. Let the notation be as above.

(i) If $K \in \mathfrak{K}(G)$, then $U^{P}=R$.

(ii) $G$ is amenable if and only if $I$ is weakly contained in $R$.

(iii) If $K \in \mathfrak{K}(G)$ is normal, $\pi: G \rightarrow G / K$ the canonical mapping and $R^{\prime}$ the regular representation of $G / K$, then $U^{J}=R^{\prime} \pi$. Also, $G / K$ is amenable if and only if $I$ is weakly contained in $U^{J}$.

Proof. (i) Clearly, ${ }_{K} U^{1}=P$ and $U^{1}=R$. Hence, by inducing in stages [2, Theorem 1], we have $U^{P}=U^{1}=R$. (ii) In view of $[6$, Theorem 3.5.2] we need only verify the reverse implication. This is proved as in the separable case [4, p. 260] making use of Lemma 4.1 of [1]. (iii) The first statement is clear. For the second, observe that the first part of Lemma 5.2 of [4] is valid for nonseparable groups as well.

The next lemma provides a slight generalization of $[6$, Theorem 2.3.3].

LEмма 2. If $K \in \mathfrak{K}(G)$, then $G$ is amenable if and only if $K$ is amenable and $I$ is weakly contained in $U^{J}$.

Proof. If $G$ is amenable, then $K$ is amenable [6, Theorem 2.3.2]. Also, $G$ acts amenably on $G / K[7$, Corollary 3.2]. Consequently, $I$ is weakly contained in $U^{J}$ by the proof of Theorem 5.1 of [7].

Conversely, if $K$ is amenable, then $J$ is weakly contained in $P$ implies $U^{J}$ is weakly contained in $U^{P}=R$, i.e. $I$ is weakly contained in $R[3$, p. 375].

2. Nets of subgroups. If $H \in \mathscr{K}(G)$, then let $\varkappa^{c}(G, H)$ (resp. $\varkappa_{c}(G, H)$ ) denote the collection of $K$ in $\mathscr{K}(G)$ which contain (resp. are contained in) $H$.

Proposition 1. $\Re_{c}(G, H)$ is closed in $\Re(G)$ and the identity mapping $\mathfrak{K}(H) \rightarrow \mathcal{K}_{c}(G, H)$ is a homeomorphism.

Proof. Since $\mathscr{K}(H)$ and $\mathscr{K}(G)$ are compact Hausdorff spaces, it suffices to show this mapping is continuous. Let $K$ in $\mathscr{K}(H)$ be the limit of a net $\left\{K_{i}\right\}$ in $\mathscr{K}(H)$. Let $\mathcal{U}(C, \mathcal{F})$ be a basic open neighborhood of $K$ in $\mathcal{K}(G)$. Then $C_{H}=C \cap H$ is a compact subset of $H$ and 
$\mathscr{F}_{H}=\{B \cap H: B \in \mathcal{F}\}$ is a finite family of nonempty relatively open subsets of $H$; in fact, $\mathcal{U}\left(C_{H}, \mathcal{F}_{H}\right)$ is an open neighborhood of $K$ in $\Re(H)$. Hence, there exists $i_{0}$ such that $i \geqq i_{0}$ implies $K_{i} \cap C \cap H=\varnothing$ and $K_{i} \cap B \cap H \neq \varnothing, B \in \mathcal{F}$. This completes the proof since $K_{i} \cap H$ $=K_{i}$, for each $i$.

Proposition 2. Let $H \in \mathfrak{K}(G)$ be normal. Then $\mathfrak{K}^{c}(G, H)$ is closed in $\Re(G)$ and the canonical bijection $\mathscr{K}(G / H) \rightarrow K^{c}(G, H)$ given by $K \rightarrow \pi^{-1}(K)$ (where $\pi: G \rightarrow G / H$ is the canonical map) is a homeomorphism.

Proof. As in the previous case, it suffices to show that the given mapping is continuous. Let $K$ in $\mathfrak{K}(G / H)$ be the limit of a net $\left\{K_{i}\right\}$ in $\mathcal{K}(G / H)$. Let $\mathfrak{U}(C, \mathfrak{F})$ be a basic open neighborhood of $\pi^{-1}(K)$ in $\mathscr{K}(G)$. Then $C^{\prime}=\pi(C)$ is a compact subset of $G / H$ and $\mathcal{F}^{\prime}=\{\pi(B)$ : $B \in \mathcal{F}\}$ is a finite family of nonempty open subsets of $G / H$; in fact, it is easy to verify that $\mathcal{u}\left(C^{\prime}, \mathcal{F}^{\prime}\right)$ is an open neighborhood of $K^{\prime}$ in $\mathscr{K}(G / H)$. Hence, there exists $i_{0}$ such that $i \geqq i_{0}$ implies $K_{i}^{\prime} \cap C^{\prime}=\varnothing$ and $K_{i}^{\prime} \cap B^{\prime} \neq \varnothing, B^{\prime} \in \mathcal{F}^{\prime}$. It is also easy to verify that this in turn implies that $\pi^{-1}\left(K_{i}\right) \in \mathcal{U}(C, \mathcal{F})$, for $i \geqq i_{0}$.

Proposition 3. If $H \in \mathfrak{K}(G)$ is open, the mapping $K \rightarrow H \cap K$ of $\mathfrak{K}(G)$ into itself is continuous.

Proof. Let $\left\{K_{i}\right\}$ be a net in $\Re(G)$ converging to $K$ in $\Re(G)$. Let $\mathcal{u}(C, \mathfrak{F})$ be a basic open neighborhood of $H \cap K$ in $\mathfrak{K}(G)$ and let $C_{H}$ and $\digamma_{H}$ be defined as in the proof of Proposition 1. Then it is clear that $\mathfrak{U}\left(C_{H}, \mathfrak{F}_{H}\right)$ is an open neighborhood of $K$. Thus, there exists $i_{0}$ such that $i \geqq i_{0}$ implies $K_{i} \in \mathcal{U}\left(C_{H}, \mathfrak{F}_{H}\right)$, i.e. $H \cap K_{i} \in \mathcal{U}(C, \mathfrak{F})$.

THeOREM 1. Let $\left\{K_{i}\right\}$ be a net in $\Re(G)$ directed by $\supseteq$, i.e. $i \geqq j$ if and only if $K_{i} \supseteq K_{j}$. Then $K_{i} \rightarrow G$ if and only if $G=\operatorname{cl}\left(U K_{i}\right)$.

Proof. Suppose $K_{i} \rightarrow G$ and $\operatorname{cl}\left(\cup K_{i}\right) \neq G$. Then there exists $x$ in $G$ such that $x \notin \operatorname{cl}\left(\cup K_{i}\right)$. Let $V$ be an open neighborhood of $x$ such that $V \cap \operatorname{cl}\left(U K_{i}\right)=\varnothing$ ( $G$ is regular). Then $\mathcal{u}(\varnothing,\{V\})$ is an open neighborhood of $G$; but for each $i, K_{i} \cap V=\varnothing$, i.e. $K_{i} \notin u(\varnothing,\{V\})$, which is a contradiction.

Conversely, suppose $G=\operatorname{cl}\left(\cup K_{i}\right)$. A basic open neighborhood of $G$ is of the form $\mathcal{U}(\varnothing, \mathcal{F})$, where $\mathcal{F}$ is arbitrary. Let $\mathcal{F}=\left\{B_{1}, \cdots, B_{n}\right\}$. Since $U K_{i}$ is dense in $G$, for each $1 \leqq k \leqq n$, there exists $i_{k}$ such that $B_{k} \cap K_{i_{k}} \neq \varnothing$. By hypothesis, there exists $i_{0}$ such that $i_{0} \geqq i_{k}, 1 \leqq k$ $\leqq n$, i.e. $K_{i_{0}} \supseteq \bigcup\left\{K_{i_{k}}: 1 \leqq k \leqq n\right\}$. Now suppose $i \geqq i_{0}$, i.e. $K_{i} \supseteq K_{i_{0}}$. Then for each $1 \leqq k \leqq n$, we have 


$$
K_{i} \cap B_{k} \supseteq \bigcup\left\{K_{i_{j}} \cap B_{j}: 1 \leqq j \leqq n\right\} \supseteq K_{i_{k}} \cap B_{k} \neq \varnothing .
$$

Thus, if $i \geqq i_{0}$, then $K_{i} \in \mathcal{U}(\varnothing, \mathcal{F})$, i.e. $K_{i} \rightarrow G$.

Corollary. If $K \in \mathfrak{K}(G)$ and $K_{i} \subseteq K$ for all $i$, then $K_{i} \rightarrow K$ if and only if $K=\operatorname{cl}\left(\bigcup K_{i}\right)$.

Proof. Apply Proposition 1.

Theorem 2. Let $\left\{K_{i}: i \in A\right\}$ be a net in $\Re(G)$ directed by $\subseteq$ i.e. $i \geqq j$ if and only if $K_{i} \subseteq K_{j}$. Then $K_{i} \rightarrow E$ if and only if $E=\cap K_{i}$.

Proof. Suppose $K_{i} \rightarrow E$. We may assume $G \neq E$. If $E \neq \cap K_{i}$, then there exists $x \in \cap K_{i}$ such that $x \neq e$. Hence, $\mathcal{U}(\{x\}, \varnothing)$ is an open neighborhood of $E$ such that for each $i \in A, x \in K_{i}$, i.e. $K_{i}$ $\notin \mathcal{E}(\{x\}, \varnothing)$, which is a contradiction.

Conversely, suppose $E=\cap K_{i}$ and let $\mathcal{U}(C, \mathfrak{F})$ be a basic open neighborhood of $E$. Then $e \notin C$ and $e \in B$ for each $B \in \mathcal{F}$. If the net $\left\{K_{i}: i \in A\right\}$ is not eventually in $\mathcal{U}(C, \mathfrak{F})$, then for each $i \in A$, there exists $i^{\prime} \in A$ such that $i^{\prime} \geqq i$ and $K_{i^{\prime}} \cap C \neq \varnothing$. Let $A^{\prime}=\left\{i^{\prime}: i \in A\right\}$. Then $A^{\prime}$ is a directed subset of $A$ and $\left\{K_{i^{\prime}}: i^{\prime} \in A^{\prime}\right\}$ is a subnet of $\left\{K_{i}: i \in A\right\}$ satisfying $K_{i^{\prime}} \cap C \neq \varnothing$, for each $i^{\prime}$. Let $x_{i^{\prime}} \in K_{i^{\prime}} \cap C$. Then $\left\{x_{i^{\prime}}: i^{\prime} \in A^{\prime}\right\}$ is a net in $C$. Since $C$ is compact, there exists a subnet $\left\{y_{j}: j \in A^{\prime \prime}\right\}$ and $x_{0} \in C$ such that $y_{j} \rightarrow x_{0}$. Let $b: A^{\prime \prime} \rightarrow A^{\prime}$ be the mapping defining this subnet. Now define $H_{j}=K_{b(j)}, j \in A^{\prime \prime}$. Hence, $y_{j}=x_{b(j)}$ is in $H_{j}$ for each $j \in A^{\prime \prime}$. It is easy to verify that $\left\{H_{j}: j \in A^{\prime \prime}\right\}$ is a subnet of $\left\{K_{i}: i \in A\right\}$ with defining map $a \circ b: A^{\prime \prime} \rightarrow A$, where $a: A^{\prime} \rightarrow A$ is the injection. We now show that $x_{0} \in \cap\left\{H_{j}: j \in A^{\prime \prime}\right\}$.

Fix arbitrary $j_{0} \in A^{\prime \prime}$. Then $b\left(j_{0}\right) \in A^{\prime}$ and there exists $j_{1} \in A^{\prime \prime}$ such that $j \geqq j_{1}$ in $A^{\prime \prime}$ implies $b(j) \geqq b\left(j_{0}\right)$ in $A^{\prime}$. But by assumption, this is equivalent to $K_{b(j)} \subseteq K_{b\left(j_{0}\right)}$, i.e. $H_{j} \subseteq H_{j_{0}}$. Let $A_{1}^{\prime \prime}=\left\{j \in A^{\prime \prime}: j \geqq j_{1}\right\}$. Then the net $\left\{y_{j}: j \in A_{1}^{\prime \prime}\right\}$ also converges to $x_{0}$. Furthermore, $j \geqq j_{1}$ implies $y_{j} \in H_{j} \subseteq H_{j_{0}}$. Thus, $x_{0}$ is an accumulation point of $H_{j_{0}}$, i.e. $x_{0} \in H_{j_{0}}$. Since $j_{0}$ was arbitrary, we have $x_{0} \in \cap\left\{H_{j}: j \in A^{\prime \prime}\right\}$.

Now let $i$ be an arbitrary element of $A$. Then there exists $j \in A^{\prime \prime}$ such that $a \circ b(j) \geqq i$ in $A$, i.e. $H_{j} \subseteq K_{i}$. Therefore, $x_{0} \in K_{i}$ which implies $x_{0}=e$ by our hypothesis. This is a contradiction since $C \cap E=\varnothing$ and the proof is complete.

Corollary. If $K \in \mathscr{K}(G)$ is normal and $K_{i} \supseteq K$ for each $i$, then $K_{i} \rightarrow K$ if and only if $K=\bigcap K_{i}$.

Proof. Apply Proposition 2.

3. Amenability. The previous results can be used to answer some 
questions on amenable subgroups. Let $\mathfrak{K}_{a}(G)$ denote the set of amenable groups in $\mathcal{K}(G)$. We will need the following:

Lemma 3. Let $\left\{K_{i}\right\}$ be a net in $\Re(G)$ and $K$ an element of $\Re(G)$ such that $K_{i} \subseteq K$ for all $i$. The following are equivalent:

(i) $K_{i} \rightarrow K$ in $K(G)$.

(ii) $\left(K_{i}, I_{i}\right) \rightarrow(K, J)$ in $\mathrm{s}(G)$.

(iii) $\left(K_{i}, R_{i}\right) \rightarrow(K, P)$ in $\delta(G)$.

Proof. In view of Lemma 2.5 of [5], we need only verify that (i) implies (ii) and (iii). The net $\left\{\left(K_{i}, K, J\right)\right\}$ converges to $(K, K, J)$ in $\mathscr{K}(G) \times S(G)$. Since restricting is continuous [5, Theorem 3.2], we see that $\left(K_{i}, J \mid K_{i}\right) \rightarrow(K, J)$ which gives (ii) since $J \mid K_{i}=I_{i}$. Similarly, the net $\left\{\left(K_{i}, E, 1\right)\right\}$ converges to $(K, E, 1)$ in $\Re(G) \times \mathfrak{S}(G)$. Since inducing is continuous $\left[5\right.$, Theorem 4.2], we have $\left(K_{i},{ }_{i} U^{1}\right)$ $\rightarrow\left(K,{ }_{K} U^{1}\right)$, which gives (iii) since $R_{i}={ }_{i} U^{1}$ and $P={ }_{K} U^{1}$.

Lemma 4. Let the notation be as in Lemma 3, but now assume that $K_{i} \supseteq K$ for all $i$. Then (i), (ii) and (iii) of Lemma 3 are again equivalent.

Proof. As before, we need only show that (i) implies (ii) and (iii). In this case, $\left(K_{i}, G, I\right) \rightarrow(K, G, I)$, so that $\left(K_{i}, I_{i}\right)=\left(K_{i}, I \mid K_{i}\right)$ $\rightarrow(K, I \mid K)=(K, J)$. Similarly, $\left(K_{i}, K, P\right) \rightarrow(K, K, P)$, so that $\left(K_{i}, R_{i}\right)=\left(K_{i},{ }_{i} U^{P}\right) \rightarrow(K, P)$.

TheOREM 3. Let $\left\{K_{i}\right\}$ be a net in $\varkappa_{a}(G)$ and $K$ an element of $\Re(G)$ such that $K \supseteq K_{i}$, for each $i$, and $K_{i} \rightarrow K$. Then $K \in \mathfrak{K}_{a}(G)$.

Proof. By Lemma 3(ii), we have $\left(K_{i}, I_{i}\right) \rightarrow(K, J)$, which implies $\left(K, K_{i}, I_{i}\right) \rightarrow(K, K, J)$ in $\mathscr{K}(G) \times \mathcal{S}(G)$. Hence, $\left(K,{ }_{\boldsymbol{\kappa}} U^{I_{i}}\right) \rightarrow(K, J)$ $\left[5\right.$, Theorem 4.2], i.e. $J$ is weakly contained in $\left\{{ }_{K} U^{I_{i}}\right\}$ as representations of $K$ (see Proposition 1.2 and Lemma 2.6 of [5]). However, since each $K_{i}$ is amenable, we also know that each $I_{i}$ is weakly contained in $R_{i}$. Thus, ${ }_{K} U^{I_{i}}$ is weakly contained in ${ }_{K} U^{R_{i}}=P$ [5, Theorem 4.3], i.e. $\left\{{ }_{K} U^{I_{i}}\right\}$ is weakly contained in $P$. Therefore, $J$ is weakly contained in $P[3$, p. 375$]$ and the proof is complete.

Corollary 1. Suppose $K \in \Re(G)$ is open and there exists a net $\left\{K_{i}\right\}$ in $\mathscr{K}_{a}(G)$ converging to $K$. Then $K \in \mathcal{K}_{a}(G)$. In particular, if $G$ is discrete, then $\mathfrak{K}_{a}(G)$ is closed in $\mathfrak{K}(G)$.

Proof. By Proposition 3 we have $K_{i} \cap K \rightarrow K$, where $K_{i} \cap K \subseteq K$ and $K_{i} \cap K \in \mathcal{K}_{a}(G)$ [6, Theorem 2.3.2]. Now apply the theorem.

Observe now that Theorem 2.3.4 of [6] is a particular case of Theorem 3. 
CoRollary 2. If $G$ is a limit of a net of amenable subgroups, then $G$ is amenable. In particular, this is the case if $G$ is a directed union of such groups.

Proof. Apply Theorem 1.

REMARK. If $G$ is nontrivial, then $\varkappa_{a}(G)$ must be nontrivial. If $x \in G$ and $x \neq e$, then the closed subgroup generated by $x$ will be abelian and thus a nontrivial element of $\mathfrak{K}_{a}(G)$.

THEOREM 4. $G$ is amenable if and only if the partially ordered set $\left(\mathcal{K}_{a}(G), \supseteq\right)$ is directed.

Proof. The forward implication is trivial. Suppose $\left(\varkappa_{a}(G), \supseteq\right)$ is directed. Then $U\left\{H: H \in \mathcal{K}_{a}(G)\right\}$ is a subgroup of $G$ whose closure $K$ belongs to $\mathcal{K}_{a}(G)$ (apply the Corollary to Theorem 1 and Theorem 3 ). Since the conjugates of $K$ must also belong to $\mathfrak{K}_{a}(G)$, it follows that $K$ must be normal. In fact, $K$ must be $G$ itself. Otherwise, $G / K$ contains a nontrivial amenable subgroup whose inverse image is an element of $\mathscr{K}_{a}(G)$ (Lemmas 1 and 2) which is strictly larger than $K$.

This theorem shows that a nonamenable group cannot contain a largest amenable subgroup. However, the next best thing is true.

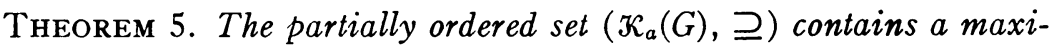
mal element.

Proof. Let $\mathscr{L}$ be a linearly ordered subset of $\mathcal{K}_{a}(G)$. Then $\mathscr{L}$ is a net directed by $\supseteq$. Hence, it must converge to $\operatorname{cl}(\cup\{L: L \in \mathfrak{L}\})=H$ which is amenable (Corollary to Theorem 1 and Theorem 3 ). $H$ is clearly an upper bound for $\mathcal{L}$. Now apply Zorn's lemma.

As an illustration of this theorem, let $G$ be the free group on two generators $a$ and $b$. Let $G_{a}$ (resp. $G_{b}$ ) denote the subgroup generated by $a$ (resp. $b$ ). Then $G_{a}$ and $G_{b}$ are amenable since they are abelian. We now show that each is a maximal amenable subgroup of $G$ (recall that $G$ is not amenable).

Let $H$ be an amenable subgroup of $G$ such that $H \supseteq G_{a}$ and $H \neq G_{a}$. Then $H$ is also free. Let $n$ be the cardinality of a set of generators for $H$. If $n \geqq 2$, then $H$ contains a copy of $G$ and cannot be amenable. Thus, $n=1$. Let $c \in G$ be a generator for $H$. Since $a \in H$, there exists an integer $q$ such that $a=c^{q}$. Since $q$ cannot be 0 or 1 , we have a nontrivial relation in $G$ which is a contradiction.

ConCluding REMARKs. It is interesting to observe that the main results of this section, Theorems 3,4 and 5, are consequences (primarily) of Theorem 1 and Lemma 3. Since Theorem 2 and Lemma 4 are natural analogues of these last two results in the opposite direc- 
tion, it seems likely that for a suitable replacement of $\mathscr{K}_{a}(G)$, Theorems 3,4 and 5 also have natural analogues in the opposite direction. We conjecture that $\mathscr{K}_{1}(G)=\left\{K \in \mathscr{K}(G): I\right.$ is weakly contained in $\left.U^{J}\right\}$ is this replacement (see $[5$, p. 442$]$ ).

\section{REFERENCES}

1. L. W. Baggett, A weak containment theorem for groups with a quotient R-group, Trans. Amer. Math. Soc. 128 (1967), 277-290. MR 36 \#3921.

2. R. J. Blattner, On induced representations, Amer. J. Math. 83 (1961), 79-98. MR 23 \#A2757.

3. J. M. G. Fell, The dual spaces of $C^{*}$-algebras, Trans. Amer. Math. Soc. 94 (1960), 365-403. MR 26 \#4201.

4. - Weak containment and induced representations of groups, Canad. J. Math. 14 (1962), 237-268. MR 27 \#242.

5. - Weak containment and induced representations of groups. II, Trans. Amer. Math. Soc. 110 (1964), 424-447. MR 28 \#3114.

6. F. P. Greenleaf, Invariant means on topological groups, Van Nostrand Math. Studies, no. 16, Van Nostrand Reinhold, New York, 1969. MR 40 \#776.

7. - Amenable actions of locally compact groups, J. Functional Analysis 4 (1969), 295-315. MR 40 \#268.

OAKLAND University, Rochester, Michigan 48063 